\title{
Biosynthesis of Gold Nanoparticles by Essential oil of Diplotaxis acris, Characterization and Antimicrobial activities
}

\author{
MOHAMED HABIB OUESLATI ${ }^{1,2 *}$ LOTFI BEN TAHAR ${ }^{1,2,3}$, \\ A. KHUZAIM ALZAHRANI ${ }^{4}$, JAMITH BASHA ${ }^{4}$ and OMAR H. ABD ELKADER ${ }^{5}$ \\ ${ }^{1}$ Northern Border University, College of Science of Arar, P.O. Box 1231, Arar 91431, \\ Kingdom of Saudi Arabia. \\ 2Université de Carthage, Faculté des Sciences de Bizerte, LR18 ES11, Laboratoire des \\ composés hétéro-organiques et des matériaux nanostructurés, 7021, Zarzouna, Tunisie. \\ ${ }^{3}$ Tunis El Manar University, Faculty of Science of Tunis, Campus Universitaire, 2092, Tunisia. \\ ${ }^{4}$ Northern Border University, College of Applied Medical Sciences, Arar, Saudi Arabia. \\ ${ }^{5}$ Physics \& Astronomy Department, Science College, King Saud University, P.O. Box 2455, \\ Riyadh, 11451, Saudi Arabia. \\ ${ }^{*}$ Corresponding author E-mail: ouesmed74@gmail.com
}

http://dx.doi.org/10.13005/ojc/370220

(Received: February 14, 2021; Accepted: March 20, 2021)

\begin{abstract}
The present work reports a green biosynthesis of gold nanoparticles (EO-AuNPs) using an Essential oil (EO) as a reducing agent of the $\mathrm{Au}(\mathrm{III})$ in $\mathrm{HAuCl}_{4}$. The $\mathrm{EO}$ was extracted by hydro-distillation from Diplotaxis acris flowers. A total of 16 compounds were detected from the EO oil by using GC-MS and 5-methylsulfanylpentanenitrile was identified as the major component $(73.60 \%)$. The biosynthesized EO-AuNPs were characterized performing UV-Vis, IR, XRD and TEM analyses. The UV-Vis revealed the typical features of surface plasmon resonance (SPR) of AuNPs at $\sim 526 \mathrm{~nm}$. The FT-IR spectrum of the biosynthesized nanoparticles exhibited the features of the nitrile $(-\mathrm{C} \equiv N)$ functional group indicating that the $-\mathrm{C} \equiv \mathrm{N}$-bearing $\mathrm{EO}$ components are likely acting as reducing and stabilizing agents for the formation of EO-AuNPs. The plausible scheme of EO-AuNPs formation was proposed. The TEM analysis showed that the EO- AuNPs were almost spherical in shape with an average particle size of $12.7 \mathrm{~nm}$. In addition, the antimicrobial activity was carried out by diffusion of agar wells method. The results proved that the EO-AuNPs displayed a potential antimicrobial against Gram-negative strains, with a maximum zone of inhibition of $16 \mathrm{~mm}$ for $E$. coli at a concentration of $100 \mu \mathrm{g} / \mathrm{mL}$.
\end{abstract}

Keywords: Essential oil, Diplotaxis acris, GC-MS, Gold nanoparticles, XRD, TEM, Antimicrobial activity.

\section{INTRODUCTION}

The field of nanoscience/nanotechnology is a field of research in full expansion which focuses on the elaboration, characterization, application and hazardous effects of new materials with unique or different properties as compared to the 'classical' counterpart materials ${ }^{1-4}$. The considerable efforts

This is an Open Access article licensed under a Creative Commons license: Attribution 4.0 International (CC- BY). Published by Oriental Scientific Publishing Company @ 2018 
made in recent years in research in the field of nanoparticles makes it possible to establish the biological properties of certain nanoparticles. Among the large number of nanoparticles developed for biomedical applications, metal nanoparticles, in particular gold nanoparticles (AuNPs) appear to be extremely attractive ${ }^{5}$. Gold is a noble metal, very little sensitive to chemical attacks and presents high biocompatibility adapted to biomedical applications ${ }^{6}$. Additionally, the gold nanoparticles have flexible optical properties depending on the size, shape and dielectric environment ${ }^{7}$. Besides, the application possibilities of AuNPs are not only related to the intrinsic properties of their metallic core but also and above all to their enormous surface capacity for the functionalization by various biomolecules ${ }^{8}$. The stability and the size of these nanoparticles in aqueous solution (for biological applications) are essentially a function of the various ligands grafted on the surface of the nanoparticles which have hydrophilic characteristics. Indeed, selected stabilizing agents such as polyphenols, proteins, polysaccharides as well as essential oil containing of the phytochemicals, were used for the synthesis of gold nanoparticles ${ }^{9-11}$. Diplotaxis acris is one among 34 species from Diplotaxis genus, belonging to the Brassicaceae (Crucifera) family ${ }^{12}$. The genus is native from North Africa to Middle East. To the best of our knowledge, the previous phytochemical work in $D$. acris plant showed, mainly, the identification of flavonoids ${ }^{13}$. The essential oil investigation of the species was not studied and this is the first report describing the profile of the volatiles from the species. Some nanoparticles show specific antimicrobial activity for bacteria, viruses or fungi without being toxic to mammalian cells ${ }^{14-16}$. Currently, the antimicrobial applications of nanoparticles range from bacteria-resistant paints, cosmetics, toothpastes, food packaging to coating metal grafts or surgical instruments. Nevertheless, the replacement of antibiotics by nanoparticles requires additional research that will evaluate the risks to humans, animals and the environment of nanoparticles whose physical and chemical properties are so innovative. In the present research work, we report the extraction and the characterization of an essential oil (EO) of Diplotaxis acris. The EO was then used to produce colloids of functionalized gold nanoparticles (EO-AuNPs). These NPs were systematically characterized by Fourier-transformed infrared (FTIR), UV-Visible spectroscopies, X-ray diffraction
(XRD) and transmission electron microscopy (TEM). The EO-AuNPs were then assessed for their antibacterial activity by the well diffusion procedure (CLSI 2017) against four clinical pathogenic bacteria. The obtained results were interpreted and compared with those reported in the literature for similar nanoparticles.

\section{MATERIALS AND METHODS}

\section{Chemicals and reagents}

All chemicals used were of analytical grade and were used as received without any further purification. Aqueous solutions were prepared with ultrapure water of conductivity less than $50 \mathrm{~S} / \mathrm{m}$.

\section{Plant material}

In March 2020, Diplotaxis acris fresh flowers were harvested during the bloom stage from wady Arar, Northern Region of Saudi Arabia. The taxonomy of the species was verified by Dr. Arbi Guetat using the reference of flora of Saudi Arabia 17 Faculty of Sciences, Northern Border University, Kingdom of Saudi Arabia.

\section{Extraction of the essential oil}

EO was extracted from flowers of Diplotaxis acris ( $1 \mathrm{~kg}$ ) by hydro-distillation method using a Clevenger's apparatus for 3 hours. The EO collected was dried over anhydrous sodium sulfate and stored at $4^{\circ} \mathrm{C}$ for further use.

\section{Oil composition analysis}

The chromatographic analysis was carried out by GC-MS (QP2010 Ultra, Shimadzu, Japan) technique. The column used is Rxi-5Sil MS $(\mathrm{L}=30 \mathrm{~m}$ $\mathrm{di}=0.25 \mathrm{~mm}$ ). The carrier gas is helium with a flow rate of $1.2 \mathrm{~mL} /$ minute. The temperature of the column is automatically programmed at $60^{\circ} \mathrm{C}$ for $5 \mathrm{~min}$, then from 60 to $200^{\circ} \mathrm{C}$ for $42 \mathrm{~min}$ and finally at $220^{\circ} \mathrm{C}$ for 5 minute. The temperatures of the injector remain constant at $230^{\circ} \mathrm{C}$ and the detector is set at $220^{\circ} \mathrm{C}$. The identification of the components was made by the comparison of their mass spectra to the NIST/ NBS, Wiley libraries.

\section{Characterization techniques}

UV-Visible spectroscopy was carried out by spectrophotometer (Jasco V-670). IR spectra were recorded using Bruker Equinox FTIR spectrometer. The structure and phase purity of the EO-AuNPs were 
examined by X-ray diffraction (XRD) using a RIGAKU Ultima IV X-ray diffractometer equipped with a Cupper $\left(\lambda_{\text {сuk }}=1.5405 / 1.5444 \AA\right)$. The average crystallite sizes are estimated using the Scherrer formula ${ }^{18}$. Transmission electron microscopy (TEM) measurement was carried out on JEM-1010 transmission electron microscope operating at $100 \mathrm{kV}$.

\section{Synthetic gold nanoparticles by essential oil}

$100 \mu \mathrm{L}$ of essential oil obtained from flowers of Diplotaxis acris was dissolved in $100 \mathrm{~mL}$ of mixture ethanol/water (50/50) solution to obtain final concentration of essential oil $1 \%(\mathrm{v} / \mathrm{v})^{19}$. Two separate preparations were achieved by adding 10 and $20 \mathrm{~mL}$ of the essential oil solution to a $\mathrm{HAuCl}_{4}$ aqueous solution ( $1 \mathrm{mM}, 10 \mathrm{~mL}$ ). Each reaction mixture was then stirred at room temperature for 30 minute. For the volume of $20 \mathrm{~mL}$ of essential oil, the preparation resulted in fast aggregation of the produced suspension and the color changed from yellow to blue. Whereas, the remaining preparation (10 $\mathrm{mL}$ of essential oil) resulted in the formation of higly stable colloid of gold nanoaprtciles (EOAuNPs) as revealed by the typical change in color from yellow to wine red ${ }^{9}$. The wine red colloid was centrifuged at $16000 \mathrm{rpm}$ for $15 \mathrm{~min}$ and washed with deionized water, air dried and then kept at $4^{\circ} \mathrm{C}$ until further use.

\section{Antibacterial activity}

The In vitro antibacterial activity of the EO-AuNPs and essential oil was assessed by the well diffusion procedure (CLSI. M100-S27) ${ }^{20}$ against four clinical pathogenic bacteria, Gram-negative strains (Escherichia coli: ATCC 25922; Klebsiella pneumonia: ATCC 700603) and Gram-positive strains (Staphylococcus aureus: ATCC 254996; Enterococcus faecalis: ATCC 29212). Briefly, the bacterial inoculum was prepared with 0.5 Macfarland standards. Muller Hinton Agar (MHA) media plates were used and wells of $6 \mathrm{~mm}$ were performed using a sterile cork borer. $100 \mu \mathrm{L}$ of the tested EO-AuNPs (2 $\mathrm{mg} / \mathrm{mL})$ and essential oil $(0.5 \mathrm{mg} / \mathrm{mL})$ prepared in DMSO were dispensed in the wells of the Petri dishes. The antibiotic ampicillin $(0.1 \mathrm{mg} / \mathrm{mL})$, was used as positive control. The plates were incubated at $37^{\circ} \mathrm{C}$ for $24 \mathrm{~h}$ and the zones of inhibition around the wells was measured manually in $\mathrm{mm}$.

\section{RESULTS AND DISCUSSION}

\section{Essential oil composition}

Several methods were used to prepare essential oils from plants such as hydro distillation, soxhlet extraction, microwave extraction and supercritical carbon dioxide extraction ${ }^{21}$. Among them, hydro distillation was used in this study because it is the most widely process in laboratories due to its simple operation and low cost. The essential oil of Diplotaxis acris flowers was obtained with yield $0.02 \%$. GC-MS analysis of the oil detected 16 chemical components listed in Table 1, which represented $99.11 \%$ of all the organic volatiles compounds. According to the Table 1, the classes of compounds in the oil were nitrogen and sulfur compounds, monoterpene hydrocarbons, and other compounds. Nitrile and sulfur compounds were found to be the most dominant class of essential oil with a percentage of $86.42 \%$, represented by 5-methylsulfanylpentanenitrile as a major constituent (73.6\%) followed by methylphenylisocyanate (9.62\%) (Fig. 1). Monoterpenes and sesquiterpenes including $\alpha$-pinene, $\beta$-pinene para cymene, $\alpha$-thujene and $\beta$-elemene were the minority products $(4.3 \%)$ represent the second class. The major product nitrile 5-methylthiopentanenitrile was also detected as a major constituent $(93 \%)$ of the essential oil of the species Diplotaxis tenuifolia ${ }^{22}$ and minor constituent $(9.2 \%)$ of the volatile fraction of Eruca vesicaria (Brassicaceae) ${ }^{23}$. Nitriles, thiocyanates and isothiocyanates metabolites produced by the hydrolysis of Glucosinolates, were known to be an important class in Brassicaceae (Cruciferous) family ${ }^{24}$.

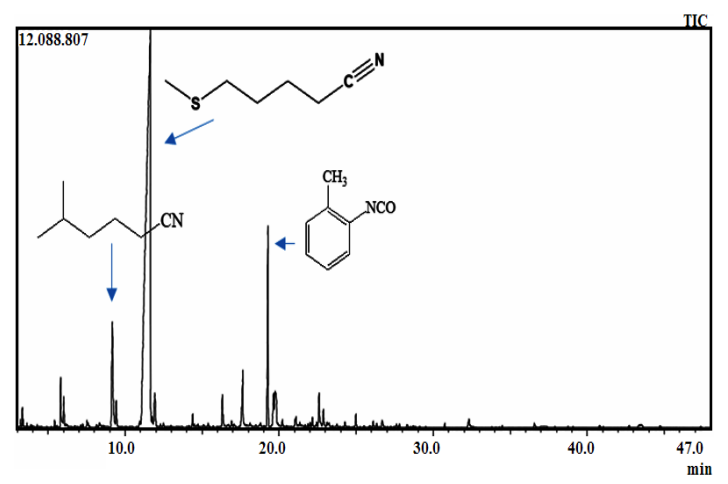

Fig. 1. GC-MS chromatogram of essential oil composition of Diplotaxis acris flowers 
Table 1: Constituents of the essential oil from the Diplotaxis acris flowers

\begin{tabular}{ccc}
\hline No & Compound & Relative amount (\%) \\
\hline 1 & 2-Isopropoxyethyl butyrate & 2.8 \\
2 & 5-methylsulfanylpentanenitrile & 73.6 \\
3 & 2-Methylphenyl isocyanate & 9.62 \\
4 & 5-methyl-hexanenitrile & 5.28 \\
5 & heptanenitrile & 1.25 \\
6 & 2,5-Dimethyltetrahydrofuran & 1.31 \\
7 & 2-Methoxy-4-vinylphenol & 0.21 \\
8 & trans-calamenene & 1.26 \\
9 & Tetralin & 0.5 \\
10 & Isoledene & 0.12 \\
11 & $\beta$-Vatirenene & 0.22 \\
12 & $\alpha$-pinene & 0.86 \\
13 & $\beta$-pinene & 0.56 \\
14 & para cymene & 0.68 \\
15 & $\alpha$-thujene & 0.64 \\
16 & $\beta$-elemene & 0.22 \\
\hline
\end{tabular}

\section{Synthesis and Characterization of EO-AuNPs}

Several previous studies have shown that AuNPs can be biosynthesized from $\mathrm{Au}(\mathrm{III})$ slats using an essential oil (EO). The EO plays a double role; it acts as a reducing as well as a stabilizing agent ${ }^{25}$. In addition, these previous studies have demonstrated that various synthesis parameters such as the, composition and the concentration of the essential oil make it possible to obtain colloids of AuNPs with controlled morphology. As a results of these approaches, we synthesized EO-AuNPs by reduction of $\mathrm{Au}^{3+}$ ions to $\mathrm{Au} 0$ with essential oil solution obtained by hydro-distillation from Diplotaxis acris flowers for the first time. To study the effect of these parameters on the EO-AuNPs synthesis reaction, we used the essential oil as a reducing agent with a modification of the concentration.

The UV-Vis absorption spectra of two the produced suspensions $\left(\mathrm{HAuCl}_{4}(1 \mathrm{mM}, 10 \mathrm{~mL})\right.$ in 10 $\mathrm{mL}$ and $20 \mathrm{~mL}$ EO solution) along with the photo of the two suspensions are shown in Figure 2.

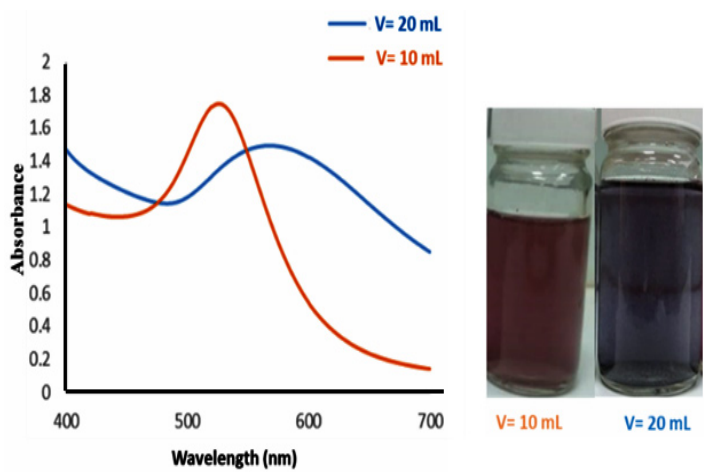

Fig. 2. UV-Visible absorption spectra of the produced suspensions (See the photo) of EO-AuNPs
The formation of EO-AuNPs was indicated by the differences in the color of the reaction mixture as shown in Fig. 2. Clear differences between the two suspension can be revealed. Indeed, as can be seen, the increasing of the volumes of $E O(1 \%)$ at $20 \mathrm{~mL}$ added to the solution of $\mathrm{AuCl}_{3}(10 \mathrm{~mL}, 1 \mathrm{M})$ resulted in macroscopic aggregation of the formed nanoparticles and a blue colored suspension. The aggregation can be confirmed by the appearance of broader surface plasmon resonance (SPR) bands at $586 \mathrm{~nm}$ in UV-Vis spectrum (Fig. 2) which could be related to an increase in the amount of bioreducing agent present in essential oil. These results also agree with previous results, suggesting the broader SPR bands indicate large AuNPs size and tend towards agglomerate formation, while narrower bands refer to the formation of AuNPs smaller in size ${ }^{26-27}$. On the other hand, the addition of only $10 \mathrm{~mL}$ of the EO (1\%) to the gold salt solution was more efficient for the biosynthesis of EO-AuNPs resulting in wine red highly stable AuNPs colloid. The band at $526 \mathrm{~nm}$ in the UV-Visible spectrum of the suspension (Fig. 2) confirmed the biosynthesis of EO-AuNPs which may be due to excitation SPR band in EO-AuNPs. The value of SPR bands was found in the range of previously biosynthesized AuNPs by various extract plants ${ }^{9-11}$. The TEM images (Fig. 3) depicted the morphology of the biosynthesized EOAuNPs. As can be seen, the produced particles are almost spherical in shape with a particle size in the range 4.4-24.4 $\mathrm{nm}$ and an average size of $12.7 \mathrm{~nm}$. The polydispersity of EO-AuNPs may be due to the presence of several reducing phytochemicals in EO. The XRD analysis (Fig. 4) shows broad diffraction peaks in the (20-80) $2 \theta$ region. The diffraction peaks at $38.1^{\circ} 44.5^{\circ} 64.6^{\circ}$ and $77.5^{\circ}$ indicating (111), (200), (220) and (311) planes, respectively, that are characteristic for cubic-face-centered (FCC) of gold crystal ${ }^{28}$. The average size calculated using the Scherrer equation is $13.16 \mathrm{~nm}$, which is a little larger than the particle size obtained from TEM. The peak values mentioned were also in agreement with the FCC structures of AuNPs obtained by green synthesis methods ${ }^{29-31}$. The biosynthesis of EO-AuNPs could be attributed to the presence of nitrile groups in 5-methylsulfanylpentanenitrile (73.60\%) and 5-methyl-hexanenitrile (5.22\%) acting as reducing and stabilizing agents. The mechanism of the formation of EO-AuNPs can be explained as 
follows (Scheme 1): First, the nitrile function forms with $\mathrm{Au}^{3+}$ ion an intermediate complex. Second, this complex can undergo further oxidation to carboxylic acid form, while $\mathrm{Au}^{3+}$ ions are reduced to elemental gold $\left(\mathrm{Au}^{0}\right)$. Finally, once the nanoparticles are formed, S, N and O, containing molecules, known to possess good affinity with AuNPs, will stabilize these nanoparticles in the form of very stable colloid.

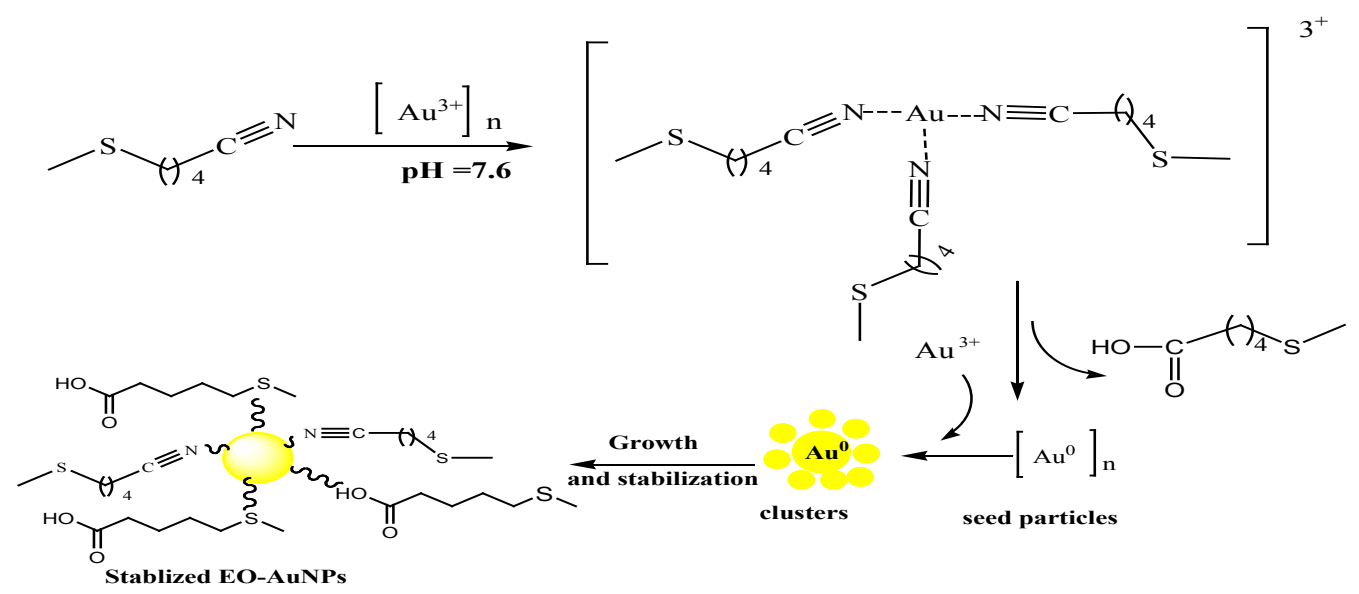

Scheme 1. Plausible mechanism of the formation of AuNPs

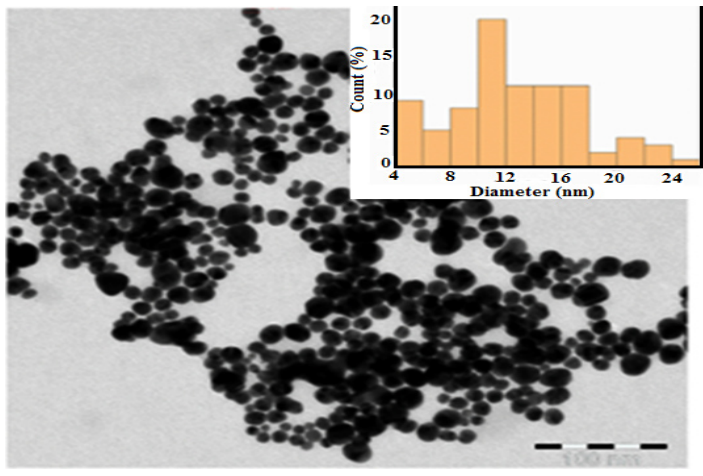

Fig. 3. TEM image of EO-AuNPs with its associated particle size distribution histogram

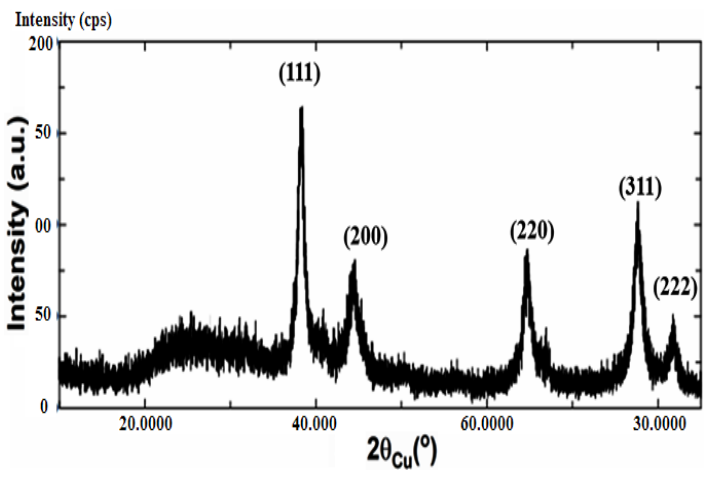

Fig. 4. XRD pattern of dried powder of biosynthesized EO-AuNPs

Figures 5, shows both the FTIR spectra of the essential oil (EO) and the synthesized EOAuNPs. A number of changes in both the position and the relative intensity of the EO in the FTIR spectrum of EO-AuNPs $(3370,2985,2946,2385$,
2282,2262, 1638.13, 1481.29, 1095, $565 \mathrm{~cm}^{-1}$ ) as compared to the FTIR spectrum of bare EO (2975, $2851,2274,2256,2286,1638,1483.5,1354.6,1245$, $\left.1080.42 \mathrm{~cm}^{-1}\right)$. The decrease in intensity absorption band of nitrile group $\left(2292 \mathrm{~cm}^{-1}\right)$ in the EO-AuNPs compared to that of the essential oil $\left(2299 \mathrm{~cm}^{-1}\right)$ and the appearance of two new bands of the carbonyl group (1652 $\mathrm{cm}^{-1}$ ) and the hydroxyl group (3452 $\mathrm{cm}^{-1}$ ) suggests the involvement of the nitrile group in the reduction process of $\mathrm{Au}^{3+}$ to $\mathrm{Au}^{0}$ as outlined in the proposed formation mechanism of EO-AuNPs (Scheme 1). The presence of the $\mathrm{CH}_{2} / \mathrm{CH}_{3}$ stretching features supports the grafting of the EO components onto the produced AuNPs.

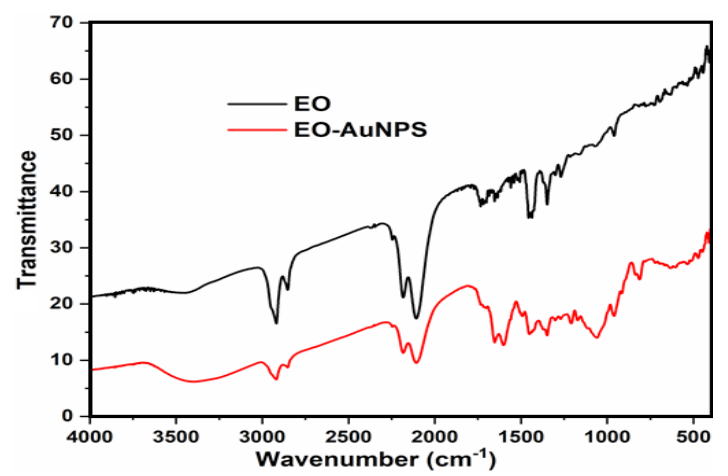

Fig. 5. The FTIR spectrum of the biosynthesized EO-AuNPs along with the that of the crude essential oil (EO)

\section{Antibacterial activity}

The In vitro antibacterial activity of biosynthesized EO-AuNPs compared to pure 
essential oil (EO) was achieved against four bacteria strains by well diffusion method. As shown in Fig. 6 , the inhibition zone values show that EO-AuNPs display higher activity against Gram-negative bacteria E. coli and K. pneumonia (inhibition zones of $16 \mathrm{~mm}$ and $14 \mathrm{~mm}$ respectively) which was higher than Gram-positive bacteria S. aureus and E. faecalis (inhibition zones of $5 \mathrm{~mm}$ and $8 \mathrm{~mm}$ respectively). This antimicrobial efficiency is closely related to the large surface area of the nanoparticles, which makes them more reactive to bacterial surfaces ${ }^{31}$. While the essential oil was active against all bacterial strains examined with zone of inhibition varying between 8 and $14 \mathrm{~mm}$. In comparison with recent studies, it was noted that EO-AuNPs exhibits superior antibacterial activity against $E$. coli, inducing a greater inhibition

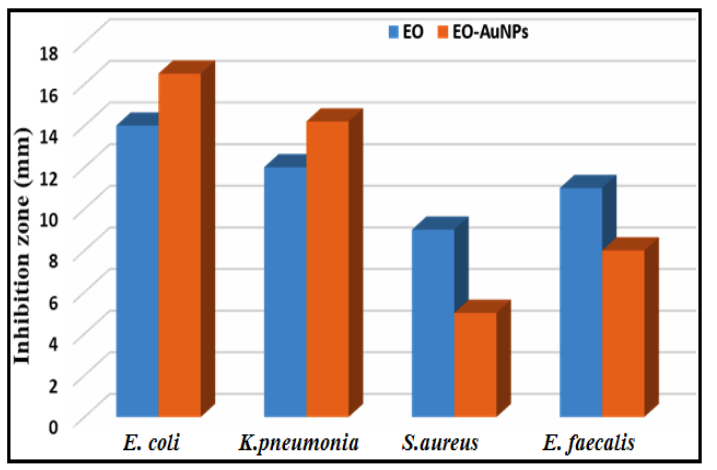

Fig. 6. In vitro antibacterial activity of the biosynthesized EO-AuNPs and the essential oil (EO) against four clinical pathogenic bacteria zone of $16 \mathrm{~mm}$ compared to AuNP synthesized from G. elongataalgal $(13.5 \mathrm{~mm})^{31}$ and almost equal to that of AuNPs synthesized from ethanolic extract of G. elongata $(17 \mathrm{~mm})^{32}$.

\section{CONCLUSION}

Phytochemical constituents of essential oil capped EO-AuNPs were produced by green chemistry route. This makes the synthesis by onestep as a fast, cost-efficient and eco-friendly process. The reduction potential of ions $\mathrm{Au}^{3+}$ to $\mathrm{Au}^{0}$ may be due to the presence of nitriles compounds with high amount in essential oil. In addition, the size of the EO-NPs produced are spherical in shape with size $12.7 \mathrm{~nm}$, which allows them to be used in biological applications. In addition, the EO-AuNPs possess good antibacterial activity in particular, against Gram-negative stains.

\section{ACKNOWLEDGMENT}

The authors are greatly indebted to the deanship of Scientific Research at Northern Border University for its funding of the present research work through the research project No. 7669-SCI2018-3-9-F.

\section{Conflict of Interest}

The authors declare that there is no conflict of interest

\section{REFERENCES}

1. Vigderman, L.; Khanal B.P,; Zubarev E. R. Functional gold nanorods: synthesis, selfassembly, and sensing applications. Adv. Mater., 2012, 24, 4811-4841.

2. Huang, X.; Neretina, S.; El-Sayed, M. A. Gold nanorods: from synthesis and properties to biological and biomedical applications. Adv. Mater., 2009, 21, 4880-4910.

3. Aderibigbe, B.A. Metal-based nanoparticles for the treatment of infectious diseases. Molecules., 2017, 22, 1370.

4. Jain, P. K.; Huang, X.; El-Sayed, I. H.; ElSayed, M. A. Noble metals on the nanoscale: optical and photothermal properties and some applications in imaging, sensing, biology, and medicine. Acc. Chem. Res., 2008, 41, 1578-1586.

5. Garrido, C.; Simpson, C. A.; Dahl, N. P.; Bresee,
J.; Whitehead, D. C.; Lindsey, E. A.; Harris, T. L.; Smith, C. A.; Carter, C. J.; Feldheim, D. L.; Melander, C.; Margolis, D. M. Gold nanoparticles to improve HIV drug delivery. Futur. Med. Chem., 2015, 7, 1097-1107.

6. Pissuwan, D.; Niidome, T.; Cortie, M. B. The forthcoming application of Au nanoparticles in drug and gene delivery systems. J. Control. Release., 2011, 149, 65-71.

7. Brown, S. D. Au Nanoparticles for the improved anticancer drug delivery of the active component of oxaliplatin, J. Am. Chem. Soc., 2010, 132, 4678-4684.

8. Oueslati, M. H.; Ben Tahar, L.; Harrath, H,; Synthesis of ultra-small gold nanoparticles by polyphenol extracted from Salvia officinalis and efficiency for catalytic reduction of p-nitrophenol and methylene blue. Green Chem Lett. Rev., 2020, 13, 18-26. 
9. Oueslati, M. H.; Ben Tahar, L.; Harrath, A. H. Catalytic, Antioxidant and Anticancer Activities of Gold Nanoparticles Synthesized by Kaempferol Glucoside from Lotus Leguminosae, Ara. J. Chem., 2020, 13, 3112-3122.

10. Lakshmanan, A.; Umamaheswari, C.; Nagarajan, N. S. A facile phyto-mediated synthesis of gold nanoparticles using aqueous extract of Momordica cochinchinensis rhizome and their biological activities, J. Nanosci. Technol., 2016, 2, 76-80.

11. Sindhu, K.; Rajaram, A.; Sreeram, K.J.; Rajaram, R.; Curcumin conjugated gold nanoparticle synthesis and its biocompatibility, RSC Adv., 2014, 4, 1808-1818.

12. Chaudhary, S. A. Flora of the Kingdom of Saudi Arabia, Ministry of Agriculture and Water, Riyadh, Saudi Arabia., 1999, 1, 691.

13. Sameh, R.; Hussein, Mona, M. M.; Mona, E.S.; Kassem, R. R.; Abdel, L.; Reda S. M. Chemosystematic significance of flavonoids isolated from Diplotaxis acris (Brassicaceae) and related taxa. Nat. Prod. Res., 2017, 31, 347-350.

14. Zharov, V. P.; Mercer, K. E.; Galitovskaya, E. N.; Smeltzer, M.S. Photothermal nanotherapeutics and nanodiagnostics for selective killing of bacteria targeted with gold nanoparticles. Biophys. J., 2006, 90, 619-627.

15. Huang, W. C.; Tsai, P. J.; Chen, Y. C. Functional gold nanoparticles as photothermal agents for selective-killing of pathogenic bacteria. Nanomedicine., 2007, 2, 777-787.

16. Gu, H.; Ho, P. L.; Tong, E.; Wang, L.; Xu, B. Presenting vancomycin on nanoparticles to enhance antimicrobial activities. Nano Lett., 2003, 3, 1261-1263.

17. Collenette, I.S. Wildflowers of Saudi Arabia. National Commission for Wildlife Conservation, Riyadh, Saudi Arabia., 1999, 799.

18. Sundararajan, B.; Kumari, B. D. R. Novel synthesis of gold nanoparticles using Artemisia vulgaris L. leaf extract and their efficacy of larvicidal activity against dengue fever vector Aedes aegypti L. J. Trace Elem.
Med. Biol., 2017, 43, 187-196.

19. Muniyappan, N.; Nagarajan, N. S. Green synthesis of gold nanoparticles using Curcuma pseudomontana essential oil, its biological activity and cytotoxicity against human ductal breast carcinoma cells T47D. J. Environ. Chem. Eng., 2014, 2, 2037-2044.

20. CLSI. M100-S27. Performance standards for antimicrobial susceptibility testing: $27^{\text {th }}$ informational supplement. Wayne, PA: Clinical and Laboratory Standards Institute., 2017.

21. Tongnuanchan, P.; Benjakul, S. Essential oils: Extraction, Bioactivities, and Their Uses for Food Preservation. J. Food. Sci., 2014, 79, 1231-1249.

22. Rodriguez, S.A.; Vela Gurovic, M.S.; Mulet, M.C.; Murray, A.P. Diplotaxis tenuifolia (L.) DC., a source of a potentially antifungal essential oil containing nitrile. Biochem. Syst. Ecol., 2006, 34, 353e355.

23. Hichri, F.; Hichri, A. O.; Mastouri, M.; Hossan, A. S. M.; Flamini, G.; Ben Jannet, H. Chemical Composition, Antibacterial, Antioxidant and in Vitro Antidiabetic Activities of Essential oils from Eruca vesicaria. Chem. Biodiversity., 2019, 16, e1900182019.

24. Blaževic, I.; Montaut, S.; Burcul, F.; Olsen, C. E.; Burow, M.; Rollin, P.; Agerbirk, N. Glucosinolate structural diversity, identification, chemical synthesis and metabolism in plants. Phytochemistry., 2020, 169, 112100.

25. Dzimitrowicz, A.; Berent, S.; Motyka, A.; Jamroz, P.; Kurcbach, K.; Sledz, W.; Pohl, P. Comparison of the characteristics of gold nanoparticles synthesized using aqueous plante extracts and natural plant essential oils of Eucalyptus globulus and Rosmarinus officinalis. Ara. J. Chem., 2019, 12, 4795-4805.

26. Klekotko, M.; Brach, K.; Olesiak-Banska, J.; Samoc, M.; Matczyszyn, K. Popcorn shaped gold nanoparticles: Plant extract-mediated synthesis, characterization and multiphoton excited luminescence properties, Mater. Chem. Phys., 2019, 229, 56-60. 
27. El-Borady, O. M.; Ayat, M. S.; Shabrawy, M. A.; Millet,P. Green synthesis of gold nanoparticles using Parsley leaves extract and their applications as an alternative catalytic, antioxidant, anticancer, and antibacterial agents. Adv. Powder Technol., 2020, 31, 4390-4400.

28. Halder, A.; Das, S.; Bera, T.; Mukherjee, A. Rapid synthesis for monodispersed gold nanoparticles in kaempferol and anti-leishmanial efficacy against wild and drug resistant strains. RSC Adv., 2017, 7, 14159-14167.

29. Khalil, M. M. H.; Ismail, E. H.; El-Magdou, F. Biosynthesis of Au nanoparticles using olive leaf extract Arab. J. Chem., 2012, 5, 431-437.

30. Biao, L.; Tan, S.; Meng, Q.; Gao, J.; Zhang, X.; Liu, Z.; Fu, Y. Green Synthesis, Characterization and Application of Proanthocyanidins-Functionalized Gold
Nanoparticles. Nanomaterials., 2018, 8, 53.

31. Liu, Z.; Zu, Y.; Fu, Y.; Meng, R.; Guo, S.; Xing, Z.; Tan, S. Hydrothermal synthesis of histidine-functionalized single-crystalline gold nanoparticles and their $\mathrm{pH}$-dependent UV absorption characteristic. Colloid Surf. B., 2010, 76, 311-316.

32. Abdel-Raouf, N.; Al-Enazi N. M.; Ibraheem, I. B. M. Green biosynthesis of gold nanoparticles using Galaxaura elongata and characterization of their antibacterial activity. Ara. J. Chem., 2017, 10, 3029-3039.

33. El-Borady, O. M.; Ayat, M.S.; Shabrawy, M. A.; Millet, P. Green synthesis of gold nanoparticles using Parsley leaves extract and their applications as an alternative catalytic, antioxidant, anticancer, and antibacterial agents. Adv. Powder Technol., 2020, 31, 4390-4400. 